\title{
Grasshopper Mandibles Functional Morphology Adaptation to Food Plants (Orthoptera: Caelifera) from the Subtropical Forest Ecosystem, Central Taiwan
}

\author{
Tzu-Fang Hsiao' ${ }^{1}$, Jeng-Tze Yang ${ }^{2,3 *}$, Ming-Yih Chen ${ }^{1}$ \\ ${ }^{1}$ Department of Life Science, National Chung Hsing University, Taiwan \\ ${ }^{2}$ Department of Entomology, National Chung Hsing University, Taiwan \\ ${ }^{3}$ Agriculture Faculty, Agriculture and Forestry University, Chitwan, Nepal \\ Email: ^jtyang@dragon.nchu.edu.tw
}

How to cite this paper: Hsiao, T.-F., Yang, J.-T. and Chen, M.-Y. (2017) Grasshopper Mandibles Functional Morphology Adaptation to Food Plants (Orthoptera: Caelifera) from the Subtropical Forest Ecosystem, Central Taiwan. Advances in Entomology, 5, 40-53.

http://dx.doi.org/10.4236/ae.2017.51004

Received: December 4, 2016

Accepted: January 20, 2017

Published: January 23, 2017

Copyright $\odot 2017$ by authors and Scientific Research Publishing Inc. This work is licensed under the Creative Commons Attribution International License (CC BY 4.0).

http://creativecommons.org/licenses/by/4.0/ (c) (7) Open Access

\begin{abstract}
This article examined 20 species of grasshoppers, belonging to six families, in Guandaushi forest ecosystem by studying the food plants and the morphology of their mandible, among which, three species are still under further identification. Based on SEM observations, the mandibles of them could be grouped into three types corresponding to their food plant: type 1 forb-feeding, the mandible with inc is or surface consists of sharp dents, and the molar area is a deep, central concavity surrounded by short ridges; type 2 grass-feeding, the incisor surface is somewhat smooth without dents, and the molar surface has long parallel grinding ridges without central concavity; type 3 obligate-feeding, the incisor is blunt, and the molar consists of ridges with a central concavity shorter than type 1 . Among the 20 species of grasshoppers in Guadaushi forest ecosystem, the ones belonging to type 1 are Traulia ornata ornata Shiraki, 1910, Xenocatantops humilis (Seville, 1839) and Coptacra sp. 3 of Catantopidae; Atractomorpha sinensis I. Bolivar, 1905 of Pygomorphidae and Erianthella formosana (Shiraki, 1910) of Eumastacidae. The species belonging to type 2 are Oxya podisma Karny, 1915, Oxyrrhepes obtuse (De Haan, 1842) of Catantopidae; Phlaeoba albonema Zheng, 1981, Phlaeoba formosana Shiraki, 1910, and Phlaeoba sinensis I. Bol., 1914of Acridae; Formosacris koshunensis (Shiraki, 1910) and Dnopherula svenhedini (Sjöstedt, 1933) of Arcypteridae and Pternoscirta sauteri (Karny, 1915), Heteropternis respondens (Walker, 1859) and Trilophidia annulata (Thunberg, 1815) of Oeipodidae. Type 3 grasshoppers are Stenocatantops splendens (Thunb., 1815), Parapodisma sp. 1, Parapodisma sp. 2, Sinopodisma kodamae (Shiraki, 1910), and Sinopodisma formosana (Shiraki, 1910) of Catantopidae.
\end{abstract}

\section{Keywords}

Grasshopper, Functional Morphology, Feeding Adaptation, Mandible Types 


\section{Introduction}

In general, grasshoppers are phytophagous and are particular in host plant specific selection. The range of host plants specificity depends on the species [1] and variously on higher categories like as generic level limited. For the specific host plant range of grasshoppers, Chapman [2] classified three categories based on the plant categories. According to Chapman [2], the polyphagous have the host plant range covering with certain preferences species, or several plant families. On the other hand, oligophagous grasshopper has the host plant range only within one single plant family. In the third category is the monophagous, which only feed on the same plant genus as their host plants. Gangwere [3] provided another classification for grasshoppers according to the systematic account of the host plants. The first one is for bivory group that feeds on broad leaves herbs; the second one is graminivory group which feed on Gramineacea grasses; and the last one is mixed herbivory group feeding on both forbs and herbs. It was also identified by Gangwere [4] that the mandibles of grasshoppers vary in different groups. This various morphology is therefore believed to be meeting the different feeding habits of different groups of grasshoppers. Three patterns of mandibles were conducted in correspondence to the feeding groups.

Mandible of the forbivory group possesses protruding teeth in the incisor area and longitudinal grooves in the molar area. It is suitable for feeding on broad leafplants. As to the graminivory group, their mandibles consist of smoothly incisor area and many longitudinal ridges in molar area. It is fitting for the long fibers in herbs. Accordingly, the herbivory group with herbivorous mandibles serves to feed on both forbs and graminis. However, these characters could only be applied to a limited level, and are not clear cut enough, for example, in the median pattern of herbivorous mandibles of grasshoppers. That is the reason why authors study for and in much detail of the functional morphology. The materials collected from the forest ecosystem in central Taiwan are therefore attempts to apply this categorization and further clarify the distinguished characters among different types of mandibles. Bernays [5] reported that the herbivory grasshoppers have the head weight-body weight ratio higher than the forbivory ones, because larger heads possessing larger mandibles and therefore larger musculatures.

While Gangwere et al. [6] used the grasshoppers collected from a Spanish island to analyze the feeding habits of grasshoppers, Kang et al. [7] also published a case from Inner Mongolia.

This study used the grasshoppers collected from one of the LTER sites in Taiwan. It was the investigation for the long term ecological monitoring for the forest ecosystem. Meanwhile, the food plant of grasshoppers is also studied. The food preference and host plant or food plant must be made sure in some ways and will be publish in the other article in near future.

Isely [8] studied 89 species of grasshopper from Texas, America, among which, 34 species are herbivory, 37 species are forbivory and 18 species mixed type. Gangwere [4] agreed with Isely [8] and pointed out that although both maxillae and labrum are also modified in accordance to feeding habits of grasshoppers, the mandible is no doubt the most significant character. 


\section{Materials and Methods}

The grasshopper community in the Heisun forest experimental station of National Chung Hsing University, Taiwan. The subtropical rain forest was one of the 5 Long Term Ecological Research (LTER) sites in Taiwan. After earthquake 921 in 1999 the research almost stops in this site. This survey finished at that time and not able to do it much more. However, the morphological adaptation of the grasshopper mandibles is worth to publish for further study interesting. the grasshopper collected by insect swept net (40 cm diameter and $60 \mathrm{~cm}$ depth) directly from the upper layer of the vegetation. Some insect collected directly by hands off.

The collected grasshoppers are preserved in $70 \%$ ethanol for application. The mandible detached from the mouth part cleaned by using ultrasonic shaker because the mandibles of grasshoppers are hard and sclerotized. Specimens were dehydrated in $80 \%$, $90 \%, 95 \%$ ethanol. The mandible is attached on the stub staged by double sticky adhesive right after the material is dried up after 95\% ethanol evaporation. The entire specimen was observed using the SEM. Details regarding the SEM method can be found in Yang et al. [9] with slight modifications.

\section{Results}

Morphology of mandibles photographed by SEM and described as followed. The following describes the feeding types of grasshoppers collected from an experimental forest in central Taiwan.

\section{Erianthella formosana (Shiraki, 1910)}

Lateral mandible (Figure 1-1) with 4 distinct incisory teeth; incisory teeth with intercalary ridge strongly prominent; intercalary teeth acute at apex obliquely; molar area typically irregularly.

Right mandible (Figure 1-2) molar area with deeply longitudinal groove, almost cross over molar area about $91 \%$; molar area with 5 short transverse ridges, $1^{\text {st }}$ ridge 0.78 as long as width of molar area, $5^{\text {th }}$ ridge 0.87 long as width of molar area; molar 1.28 times longer than wide, about 1.35 times longer than length of incisory area.

\section{Phlaeoba formosana (Shiraki, 1910)}

Left mandible (Figure 2-1) with 4 indistinct incisory teeth; mosaic surface smooth,
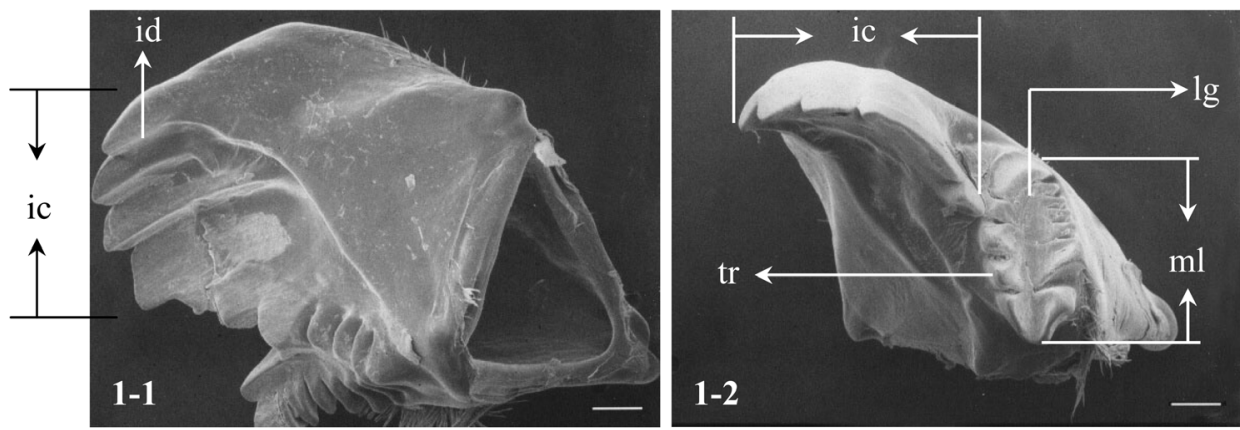

Figure 1. Erianthella formosana (Shiraki, 1910), adult mandible (type I), 1-1: left mandible posterior view; 1-2: right mandible lateral view (scale bar $=250 \mu \mathrm{m})$. a: articulation; am: abductor muscle; tr: transverse ridge; lg: longitudinal groove; ic: incisor area; ict: incisor teeth; id: intercalary edge; it: intercalary teeth; ml: molar area. 

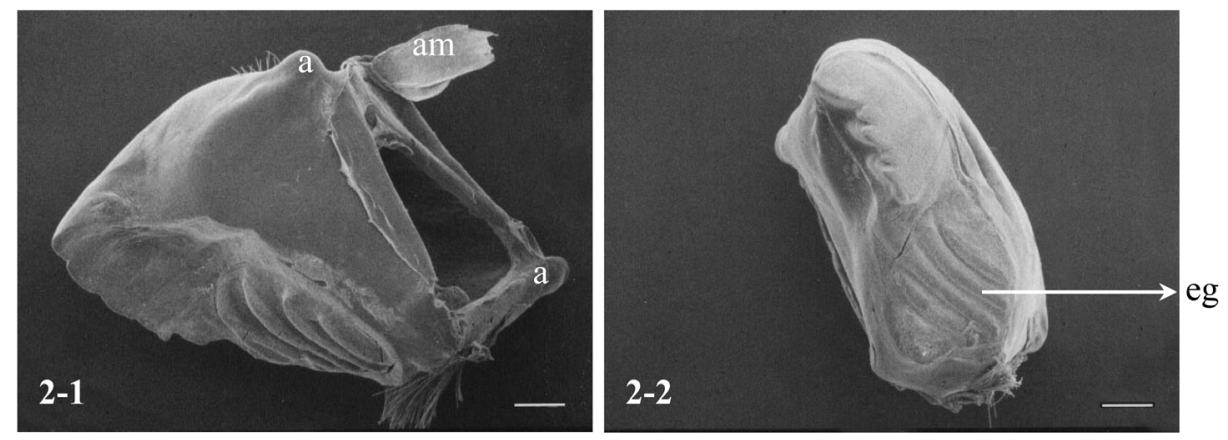

Figure 2. Phlaeoba formosana (Shiraki, 1910), adult mandible (type II), 2-1: left mandible posterior view; 2-2: right mandible lateral view (scale bar $=250 \mu \mathrm{m})$. a: articulation; am: abductor muscle; tr: transverse ridge; lg: longitudinal groove; ic: incisor area; ict: incisor teeth; id: intercalary edge; it: intercalary teeth; ml: molar area.

incisory teeth without intercalary ridge strongly prominent; intercalary teeth roundly arched at apex; molar area smooth.

Right mandible (Figure 2-2) molar area without longitudinal groove; molar area with 5 long ridges, not interrupted by longitudinal groove, $1^{\text {st }}$ ridge 0.61 times as long as width of molar area, $5^{\text {th }}$ ridge 0.56 times as long as width of molar area; molar 1.06 times longer than wide, about 1.23 times longer than length of incisory area.

\section{Sinopodisma formosana (Shiraki, 1910)}

Left mandible (Figure 3-1) with 4 distinct incisory teeth, digital like, round at apex; mosaic surface with intercalary ridge complete, strongly prominent; intercalary teeth truncate at apex; molar area irregular typically.

Right mandible (Figure 3-2) molar area with longitudinal groove, short about 0.45 times of length of molar area, groove not cross over molar area, shallow in depth; molar area with 5 long ridges, only $4^{\text {th }}$ and $5^{\text {th }}$ ones interrupted by longitudinal groove, $1^{\text {st }}$ ridge 0.65 times as long as width of molar area, $5^{\text {th }}$ ridge 0.65 times as long as width of molar area; molar 1.43 times longer than wide, about 1.23 times longer than length of incisory area.

\section{Xenocatantops humilis (Seville, 1839)}

Left mandible (Figure 4-1) with 4 indistinct incisory teeth, long plate like, truncate at apex; mosaic surface with intercalary ridge complete, strongly prominent; intercalary teeth truncate at apex; molar area irregular typically.

Right mandible (Figure 4-2) molar area with longitudinal groove deeply, short about 0.84 times of length of molar area; molar area with 5 short ridges, longitudinal groove formed shallow concaved area, $1^{\text {st }}$ ridge 0.84 times as long as width of molar area, $5^{\text {th }}$ ridge 0.63 times as long as width of molar area; molar 0.51 times longer than wide, about 0.93 times longer than length of incisory area.

\section{Atractomorpha sinensis (I. Bolivar, 1905)}

Left mandible (Figure 5-1) with 4 distinct incisory teeth, digital like; mosaic surface with intercalary ridge complete, strongly prominent; intercalary teeth truncate at apex; molar area irregular typically.

Right mandible (Figure 5-2) molar area with longitudinal groove deeply, almost cross over molar area, about 0.89 times of length of molar area; molar area with 5 short 

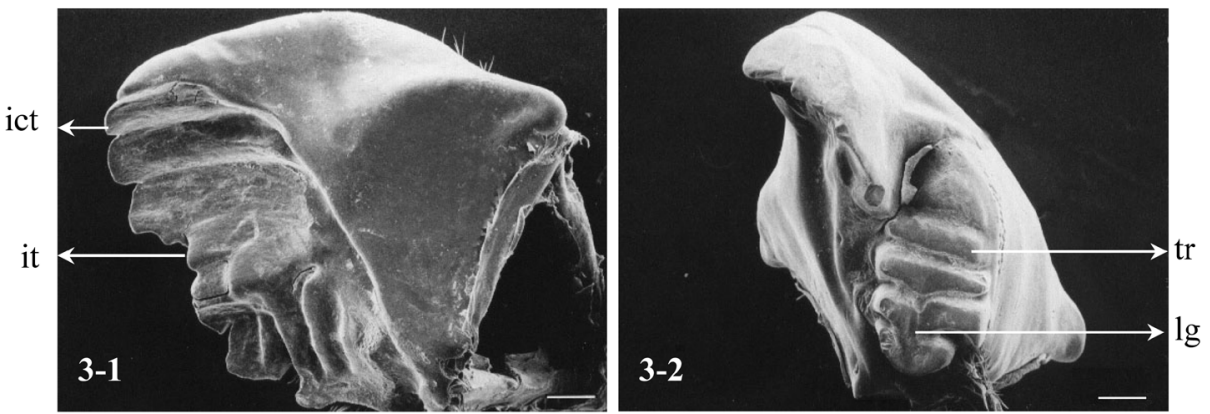

Figure 3. Sinopodisma formosana (Shiraki, 1910) adult mandible (type III), 3-1: left mandible posterior view; 3 - 2: right mandible lateral view (scale bar $=250 \mu \mathrm{m})$. a: articulation; am: abductor muscle; tr: transverse ridge; lg: longitudinal groove; ic: incisor area; ict: incisor teeth; id: intercalary edge; it: intercalary teeth; ml: molar area.
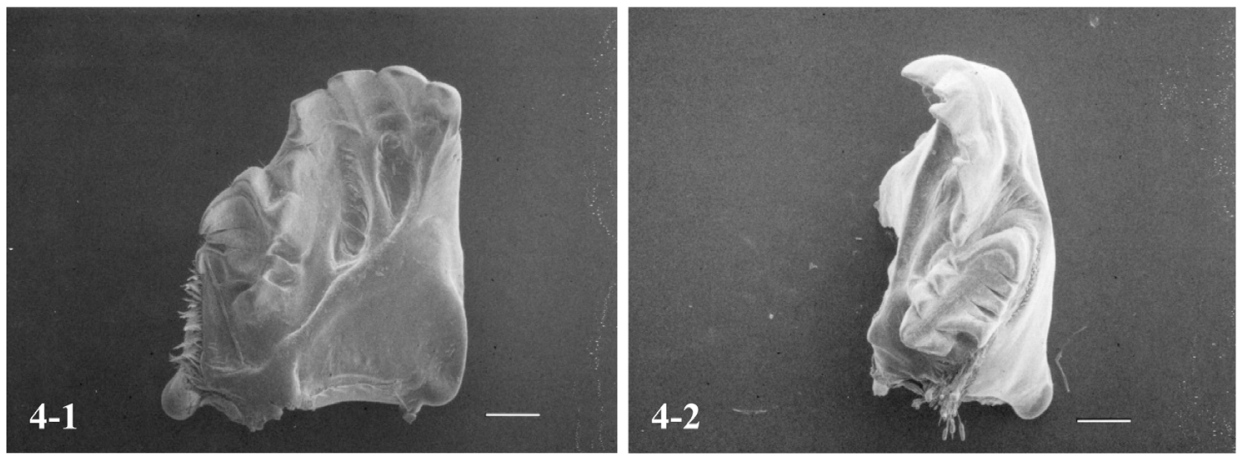

Figure 4. Xenocatantops humilis (Seville, 1839) adult mandible, 4-1: left mandible posterior view; 4-2: right mandible lateral view (scale bar $=333 \mu \mathrm{m}$ ).
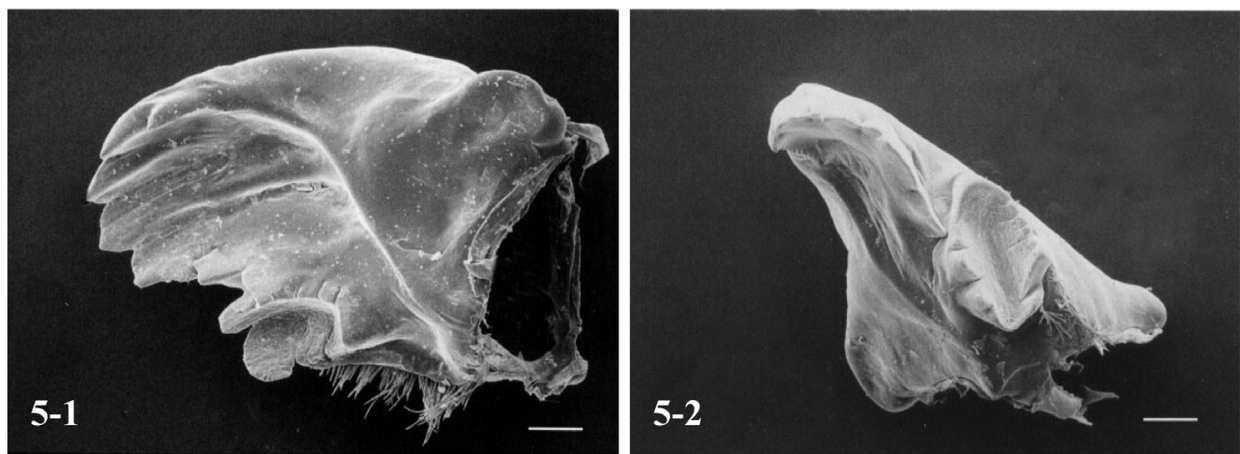

Figure 5. Atractomorpha sinensis I. Bolivar, 1905 adult mandible, 5-1: left mandible posterior view, 5-2: right mandible lateral view (scale bar $=200 \mu \mathrm{m}$ ).

ridges, longitudinal groove formed shallow concaved area, $1^{\text {st }}$ ridge 0.67 times as long as width of molar area, $5^{\text {th }}$ ridge 0.57 times as long as width of molar area; molar 1.96 times longer than wide, about 1.08 times longer than length of incisory area.

\section{Coptacra sp. 3}

Left mandible (Figure 6-1) similar to Atractomorpha sinensis with 4 distinct incisory teeth, digital like; mosaic surface with intercalary ridge complete, strongly prominent; intercalary teeth truncate at apex; molar area with cone like processes irregular typically. 

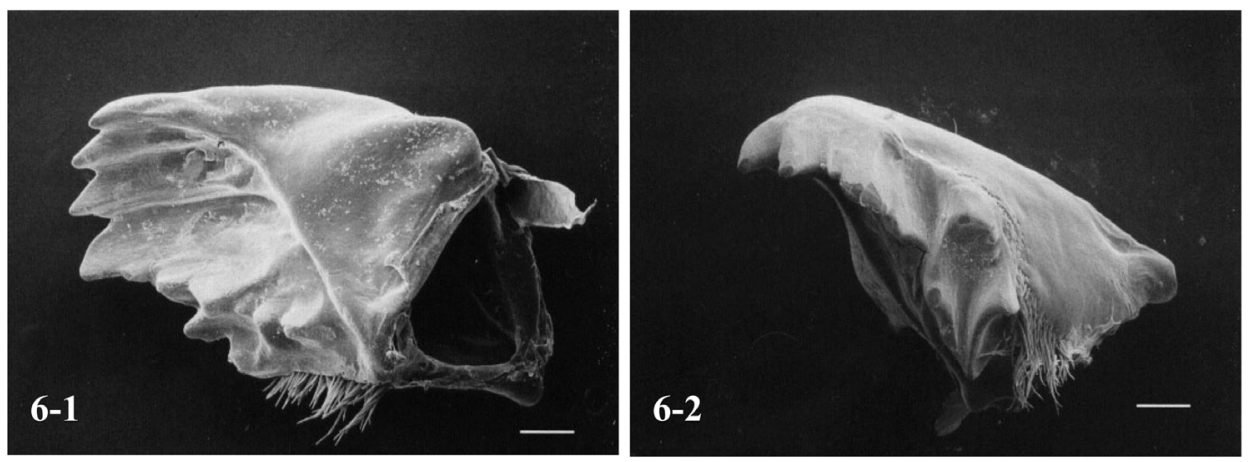

Figure 6. Coptacra sp. 3 adult mandible, 6-1: left mandible posterior view; 6-2: right mandible lateral view $($ scale bar $=200 \mu \mathrm{m})$.

Right mandible (Figure 6-2) molar area with longitudinal groove deeply, formed shallow concaved area about 0.80 times of length of molar area; molar area without recognizable ridge; molar 2 times longer than wide, about 1.05 times longer than length of incisory area.

\section{Traulia ornata ornata (Shiraki, 1910)}

Lateral mandible (Figure 7-1) is similar to Erianthella formosanas incisory area with 4 distinct incisory teeth; incisory teeth with intercalary ridge strongly prominent; intercalary teeth acute at apex middly; molar area typically irregular.

Right mandible (Figure 7-2) similar to Sinopodisma formosana molar area with longitudinal groove deeply, short about 0.68 times of length of molar area, groove not cross over molar area; molar area with 5 long ridges, $3^{\text {rd }}, 4^{\text {th }}$ and $5^{\text {th }}$ ones interrupted by longitudinal groove, $1^{\text {st }}$ ridge 0.80 times as long as width of molar area, $5^{\text {th }}$ ridge 0.60 times as long as width of molar area; molar 1.85 times longer than wide, about 1.10 times longer than length of incisory area.

\section{Stenocatantops splendens (Thunb., 1815)}

Left mandible (Figure 8-1) similar to Atractomorpha sinensis with 4 distinct incisory teeth, digital like, roundly acute at apex; mosaic surface with intercalary ridge complete, strongly prominent; intercalary teeth acute at apex; molar area with one cone like and two plate like processes arranged in line.

Right mandible (Figure 8-2) similar to Traulia ornata ornata molar area with longitudinal groove moderately deep, short about 0.43 times of length of molar area, groove not cross over molar area; molar area with 5 long ridges, $4^{\text {th }}$ and $5^{\text {th }}$ ones interrupted by longitudinal groove, $1^{\text {st }}$ ridge 0.56 times as long as width of molar area, $5^{\text {th }}$ ridge 0.60 times as long as width of molar area; molar 1.67 times longer than wide, about 1.12 times longer than length of incisory area.

\section{Sinopodisma kodamae (Shiraki, 1910)}

Lateral mandible (Figure 9-1) similar to Erianthella formosanas incisory area with 4 distinct incisory teeth, acute at apex, medially; incisory teeth with intercalary ridge strongly prominent; intercalary teeth acute round at apex; molar area with 3 broadplate like processes.

Right mandible (Figure 9-2) similar to Stenocatantops splendens and Traulia ornata ornata molar area with longitudinal groove, broad, moderately deep, short about 0.59 

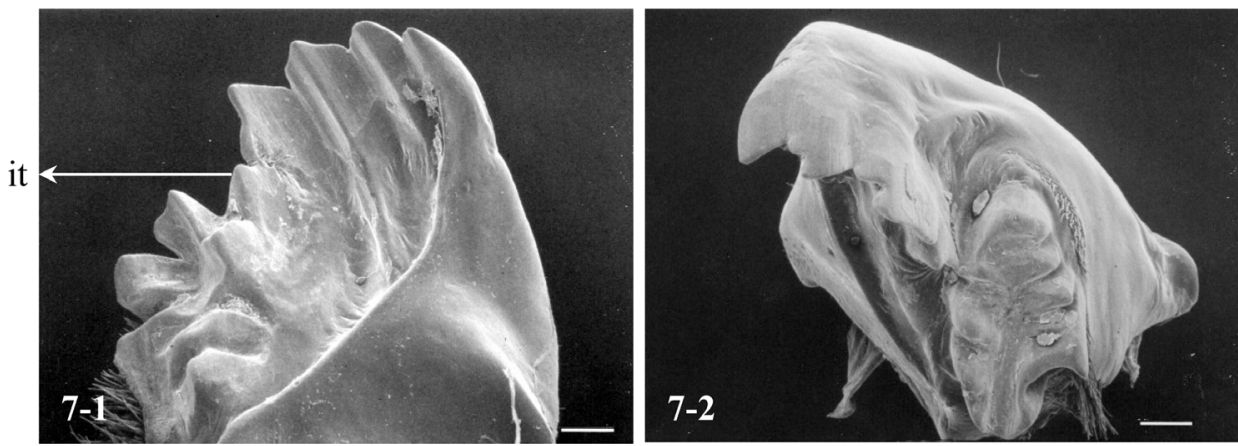

Figure 7. Traulia ornata ornate Shiraki, 1910 adult mandible, 7-1: left mandible, posterior view; 7-2: right mandible lateral view (scale bar $=250 \mu \mathrm{m})$. it: intercaray teeth.
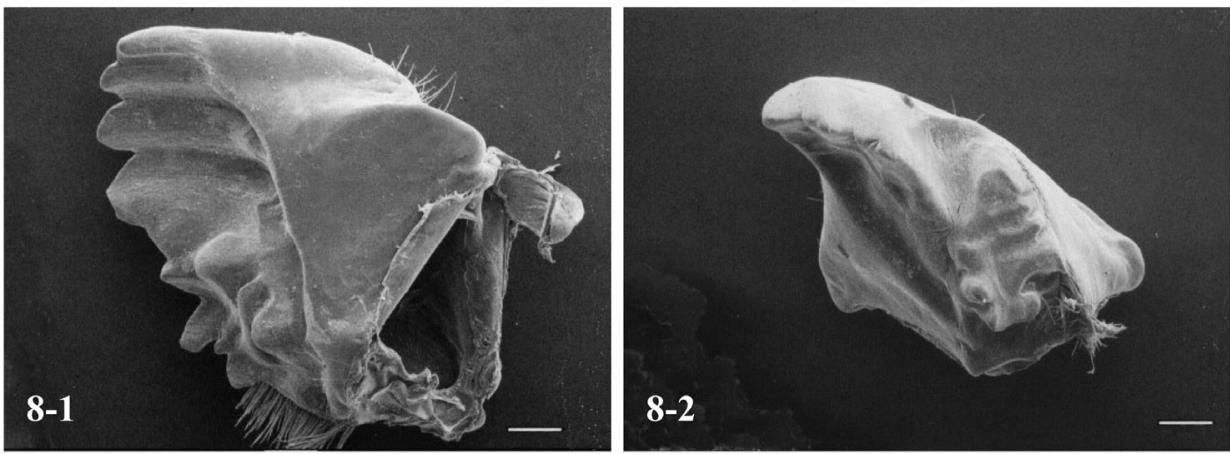

Figure 8. Stenocatantops splendens (Thunb., 1815) adult mandible, 8-1: left mandible posterior view; 8-2: right mandible lateral view (scale bar $=250 \mu \mathrm{m}$ ).
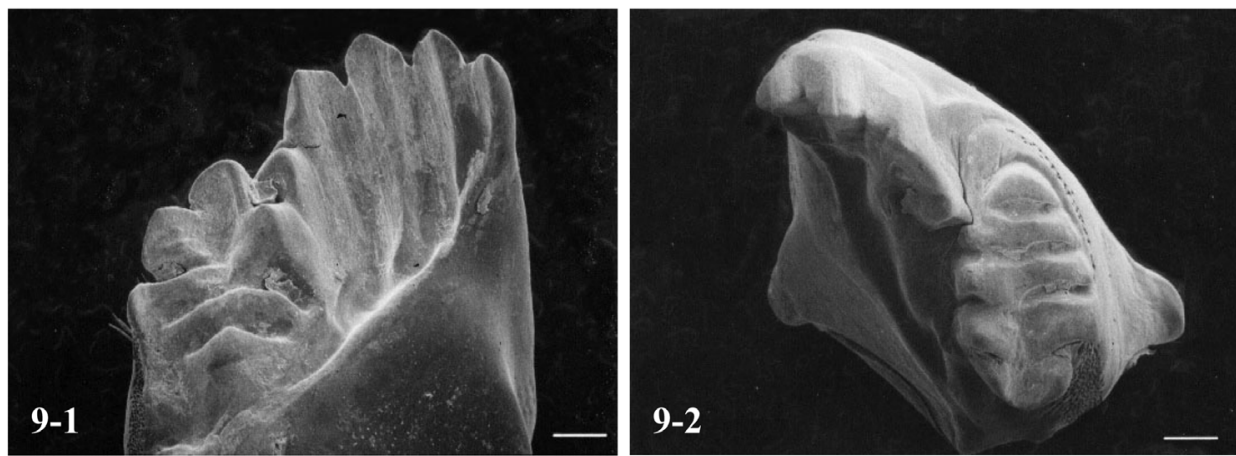

Figure 9. Sinopodisma kodamae (Shiraki, 1910) adult mandible, 9-1: left mandible posterior view; 9-2: right mandible lateral view (scale bar $=250 \mu \mathrm{m})$.

times of length of molar area, groove not cross over molar area; molar area with 5 long ridges, $4^{\text {th }}$ and $5^{\text {th }}$ ones interrupted by longitudinal groove, $1^{\text {st }}$ ridge 0.64 times as long as width of molar area, $5^{\text {th }}$ ridge 0.64 times as long as width of molar area; molar 1.82 times longer than wide, about 1.02 times longer than length of incisory area.

\section{Parapodisma sp. 1}

Lateral mandible (Figure 10-1) similar to Erianthella formosanas and Sinopodisma kodamae incisory area with 4 distinct incisory teeth, truncate at apex; incisory teeth with intercalary ridge strongly prominent; intercalary teeth obtusely acute at apex; molar area with 3 indistinct processes. 

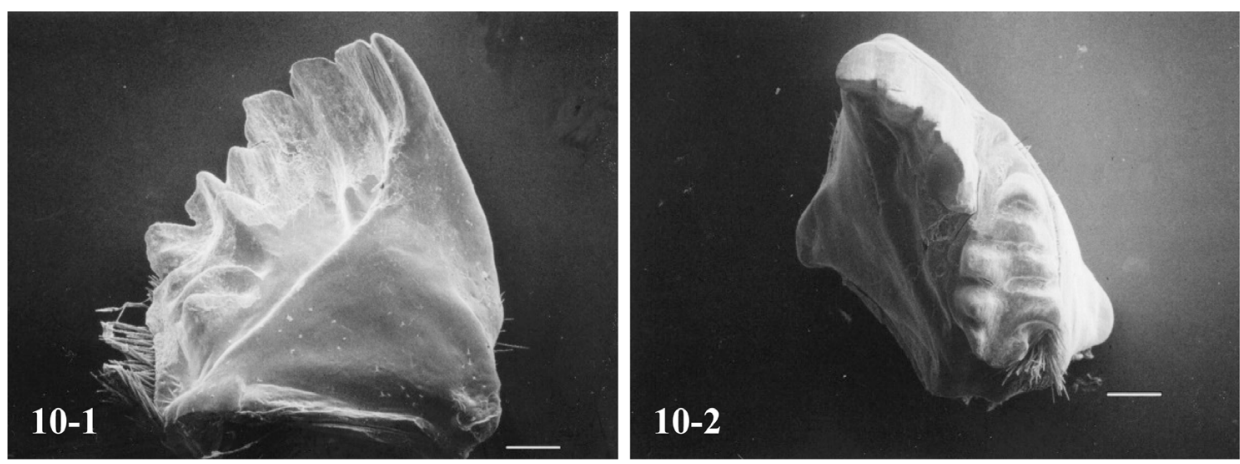

Figure 10. Parapodisma sp. 1adult mandible, 10-1: left mandible posterior view; 10-2: right mandible lateral view (scale bar $=250 \mu \mathrm{m})$.

Right mandible (Figure 10-2) similar to Stenocatantops splendens and Sinopodisma kodamae molar area with longitudinal broad groove moderately deep, short about 0.57 times of length of molar area, groove not cross over molar area; molar area with 5 long ridges, $3^{\text {rd }}, 4^{\text {th }}$ and $5^{\text {th }}$ ones interrupted by longitudinal groove, $1^{\text {st }}$ ridge 0.63 times as long as width of molar area, $5^{\text {th }}$ ridge 0.66 times as long as width of molar area; molar 2 times longer than wide, about 1.05 times longer than length of incisory area.

\section{Parapodisma sp. 2}

Lateral mandible (Figure 11-1) similar to Erianthella formosanas and Sinopodisma kodamae incisory area with 4 distinct incisory teeth, round acute at apex; incisory teeth with intercalary ridge strongly prominent; intercalary teeth truncate at apex; molar area moderate long stripe like processes.

Right mandible (Figure 11-2) similar to Stenocatantops splendens and Sinopodisma kodamae molar area with longitudinal broad groove moderately deep, short about 0.57 times of length of molar area, groove not cross over molar area; molar area with 5 long ridges, $3^{\text {rd }}, 4^{\text {th }}$ and $5^{\text {th }}$ ones interrupted by longitudinal groove, $1^{\text {st }}$ ridge 0.59 times as long as width of molar area, $5^{\text {th }}$ ridge 0.59 times as long as width of molar area; molar 1.92 times longer than wide, about long as length of incisory area.

\section{Oxya podisma (Karny, 1915)}

Left mandible (Figure 12-1) with 4 indistinct incisory teeth, round at apex; mosaic surface smooth, incisory teeth with intercalary ridge strongly prominent; intercalary teeth almost as long as incisory teeth, truncate at apex; molar area with 4 long stripe processes.

Right mandible (Figure 12-2) molar area without longitudinal groove; molar area with 6 long ridges, not interrupted by longitudinal groove, $1^{\text {st }}$ ridge 0.62 times as long as width of molar area, $6^{\text {th }}$ ridge 0.56 times as long as width of molar area; molar 1.05 times longer than wide, about 1.35 times longer than length of incisory area.

\section{Oxyrrhepes obtuse (De Haan, 1842)}

Left mandible (Figure 13-1) with 4 - 5 not recognizable incisory teeth, almost formed plate like; mosaic surface smooth, incisory teeth without intercalary ridge strongly prominent; intercalary teeth indistinct, as long as incisory teeth, truncate at apex; molar area with 5 long stripe like processes.

Right mandible (Figure 13-2) molar area sub-elliptical, without longitudinal groove; molar area with 5 long ridges, not interrupted by longitudinal groove, $1^{\text {st }}$ ridge 0.48 

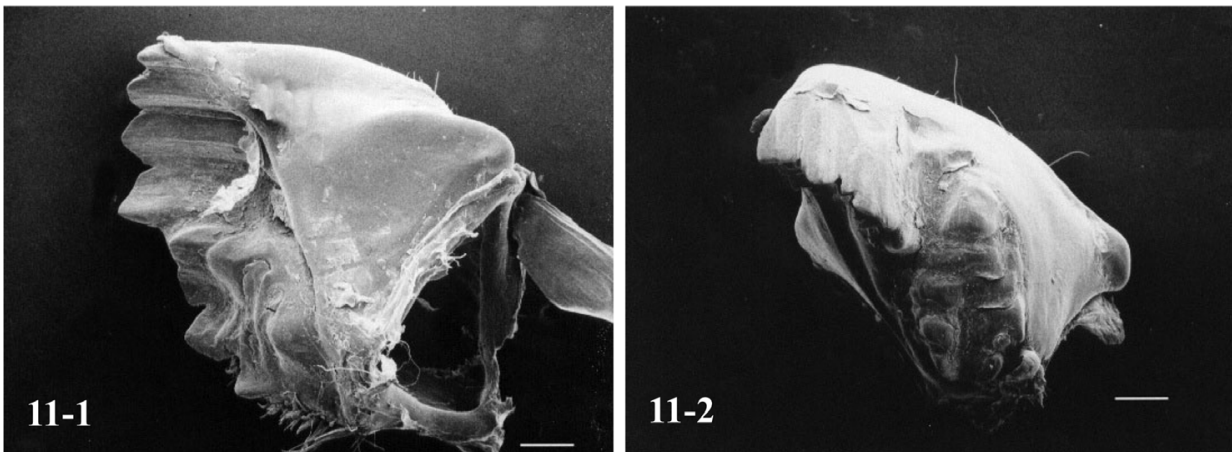

Figure 11. Parapodisma sp. 2 adult mandible, 11-1: left mandible posterior view; 11-2: right mandible lateral view (scale bar $=333 \mu \mathrm{m})$.
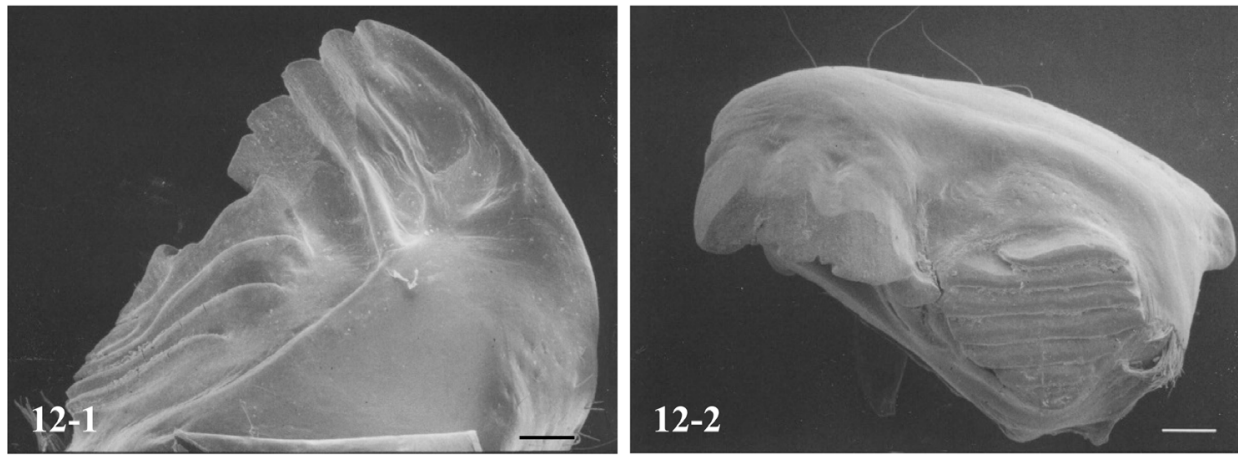

Figure 12. Oxya podisma (Karny, 1915) adult mandible, 12-1: left mandible posterior view; 12-2: right mandible lateral view (scale bar $=286 \mu \mathrm{m})$.
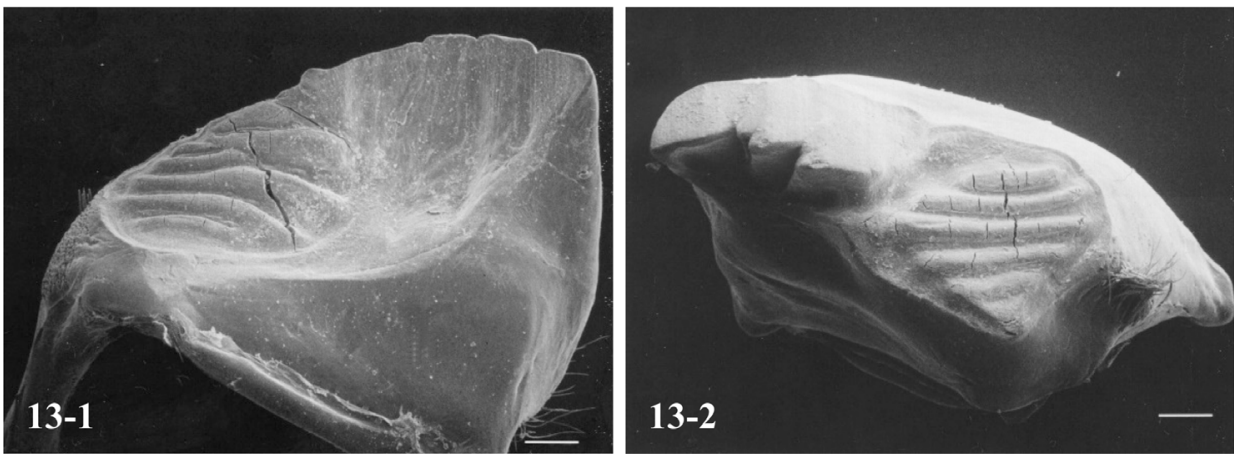

Figure 13. Oxyrrhepes obtuse (De Haan, 1842) adult mandible, 13-1: left mandible posterior vie; 13-2: right mandible lateral view (scale bar $=250 \mu \mathrm{m})$.

times as long as width of molar area, $5^{\text {th }}$ ridge 0.40 times as long as width of molar area; molar 1.06 times longer than wide, about 1.32 times longer than length of incisory area.

Phlaeoba sinensis (I. Bol., 1914)

Left mandible (Figure 14-1) with 4 incisory teeth not recognizable, almost formed plate like; mosaic surface smooth, incisory teeth without intercalary ridge; intercalary teeth indistinct, slightly shorter than incisory teeth, truncate at apex; molar area with 4 long stripe like processes.

Right mandible (Figure 14-2) molar area subquadrate, without longitudinal groove; molar area with 5 long ridges, not interrupted by longitudinal groove, $1^{\text {st }}$ ridge 0.66 

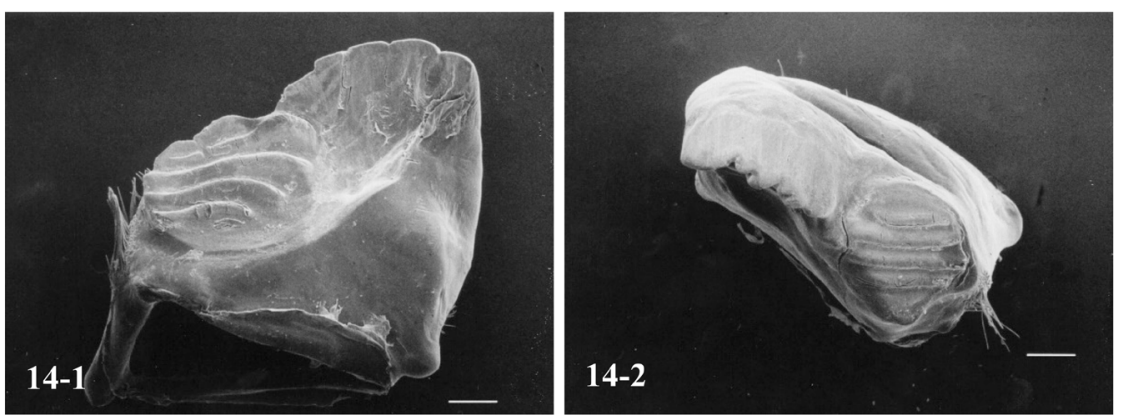

Figure 14. Phlaeoba sinensis (I. Bol., 1914) adult mandible, 14-1: left mandible posterior view; 14-2: right mandible lateral view (scale bar $=250 \mu \mathrm{m})$.

times as long as width of molar area, $5^{\text {th }}$ ridge 0.41 times as long as width of molar area; molar 1.10 times longer than wide, about 1.37 times longer than length of incisory area.

\section{Phlaeoba albonema (Zheng, 1981)}

Left mandible (Figure 15-1) with 4 incisory teeth not recognizable, almost formed plate like; mosaic surface smooth, incisory teeth truncate at apex, without intercalary ridge; intercalary teeth indistinct, slightly shorter than incisory teeth, truncate at apex; molar area with 5 long stripe like processes.

Right mandible (Figure 15-2) molar area subquadrate, without longitudinal groove; molar area with 5 long ridges, not interrupted by longitudinal groove, $1^{\text {st }}$ ridge 0.77 times as long as width of molar area, $5^{\text {th }}$ ridge 0.52 times as long as width of molar area; molar 1.10 times longer than wide, about 1.45 times longer than length of incisory area.

\section{Heteropternis respondens (Walker, 1859)}

Left mandible (Figure 16-1) with 4 incisory teeth not recognizable, almost fused and formed plate like; mosaic surface smooth, incisory teeth without intercalary ridge; intercalary teeth indistinct, slightly shorter than incisory teeth, truncate at apex; molar area with 3 layers prominent arranged in concentric triangle.

Right mandible (Figure 16-2) molar area oblong, without longitudinal groove; molar area with 5 long ridges, not interrupted by longitudinal groove, $1^{\text {st }}$ ridge 0.73 times as long as width of molar area, $5^{\text {th }}$ ridge 0.33 times as long as width of molar area; molar 1.03 times longer than wide, about 1.19 times longer than length of incisory area.

\section{Pternoscirta sauteri (Karny, 1915)}

Left mandible (Figure 17-1) with 4 incisory teeth not recognizable, almost formed plate like; mosaic surface smooth, incisory teeth truncate at apex, without intercalary ridge; intercalary teeth indistinct, shorter than incisory teeth, round acute at apex; molar area with 4 long stripe like processes, first one incomplete.

Right mandible (Figure 17-2) molar area oblong, without longitudinal groove; molar area with 5 long ridges, not interrupted by longitudinal groove, $1^{\text {st }}$ ridge 0.79 times as long as width of molar area, $5^{\text {th }}$ ridge 0.32 times as long as width of molar area; molar 1.16 times longer than wide, about 1.21 times longer than length of incisory area.

\section{Trilophidia annulata (Thunberg, 1815)}

Left mandible (Figure 18-1) with 4 incisory teeth not recognizable, almost formed plate like; mosaic surface smooth, incisory teeth truncate at apex, without intercalary ridge; intercalary teeth indistinct, shorter than incisory teeth, round at apex; molar area with 3 long stripe like processes. 

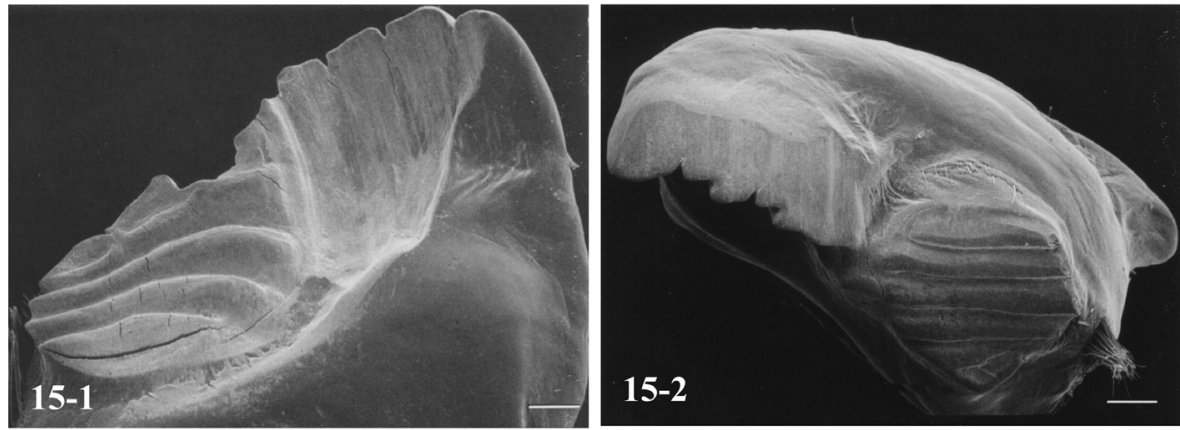

Figure 15. Phlaeoba albonema (Zheng, 1981) adult mandible, 15-1: left mandible posterior view; 15-2: right mandible lateral view (scale bar $=250 \mu \mathrm{m})$.
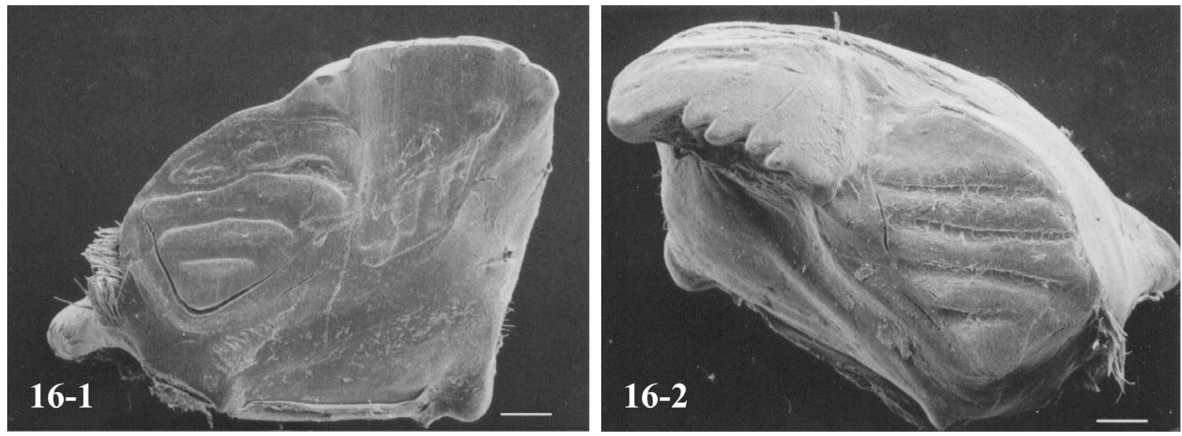

Figure 16. Heteropternis respondens (Walker, 1859) adult mandible, 16-1: left mandible posterior view; 16-2: right mandible lateral view (scale bar $=250 \mu \mathrm{m})$.
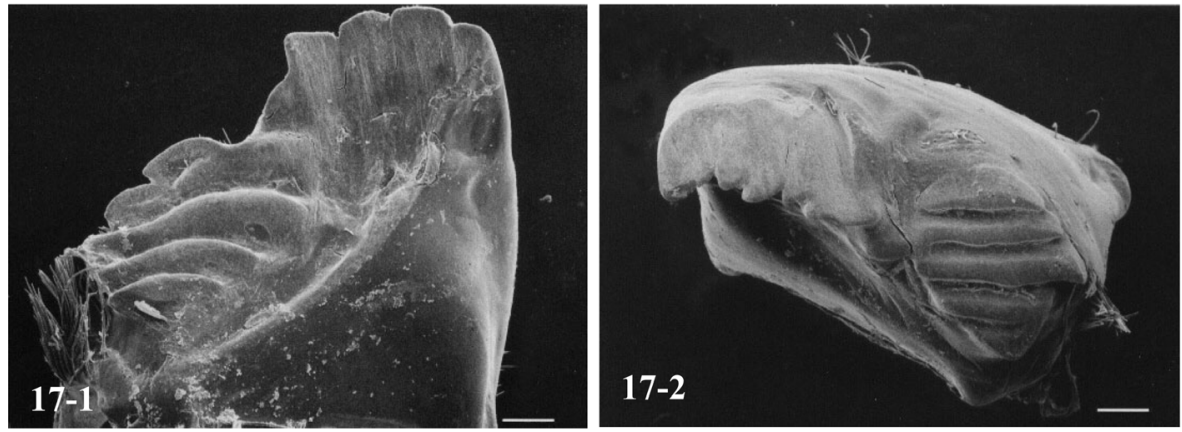

Figure 17. Pternoscirta sauteri (Karny, 1915) adult mandible, 17-1: left mandible posterior view; 17-2: right mandible lateral view (scale bar $=200 \mu \mathrm{m})$.
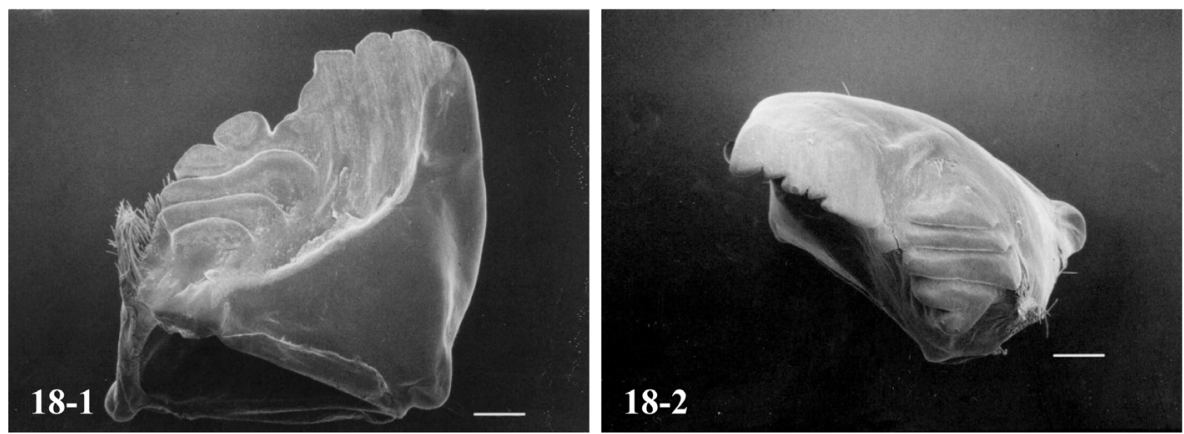

Figure 18. Trilophidia annulata (Thunberg, 1815) adult mandible, 18-1: left mandible posterior view; 18-2: right mandible lateral view (scale bar $=250 \mu \mathrm{m}$ ). 
Right mandible (Figure 18-2) molar area oblong, without longitudinal groove; molar area with 5 long ridges, not interrupted by longitudinal groove, $1^{\text {st }}$ ridge 0.70 times as long as width of molar area, $5^{\text {th }}$ ridge 0.37 times as long as width of molar area; molar 1.19 times longer than wide, about 1.19 times longer than length of incisory area.

\section{Dnopherula svenhedini (Sjöstedt, 1933)}

Left mandible (Figure 19-1) with 4 incisory teeth not recognizable, almost fused and formed plate like; mosaic surface smooth, incisory teeth without intercalary ridge; intercalary teeth indistinct; molar area with 2 long stripe like processes.

Right mandible (Figure 19-2) molar area transverse long, without longitudinal groove; molar area with 5 long ridges, not interrupted by longitudinal groove, $1^{\text {st }}$ ridge 0.52 times as long as width of molar area, $5^{\text {th }}$ ridge 0.36 times as long as width of molar area; molar 1.24 times longer than wide, about 1.30 times longer than length of incisory area.

\section{Formosacris koshunensis (Shiraki, 1910)}

Left mandible (Figure 20-1) with incisory teeth not recognizable, almost fused and formed plate like; mosaic surface smooth, incisory teeth truncate at apex, without intercalary ridge; intercalary teeth indistinct; molar area with 4 long stripe like processes.

Right mandible (Figure 20-2) with molar area oblong, without longitudinal groove; molar area with 5 long ridges, not interrupted by longitudinal groove, $1^{\text {st }}$ ridge 0.59 times as long as width of molar area, $5^{\text {th }}$ ridge 0.41 times as long as width of molar area; molar 1.05 times longer than wide, about 1.08 times longer than length of incisory area.
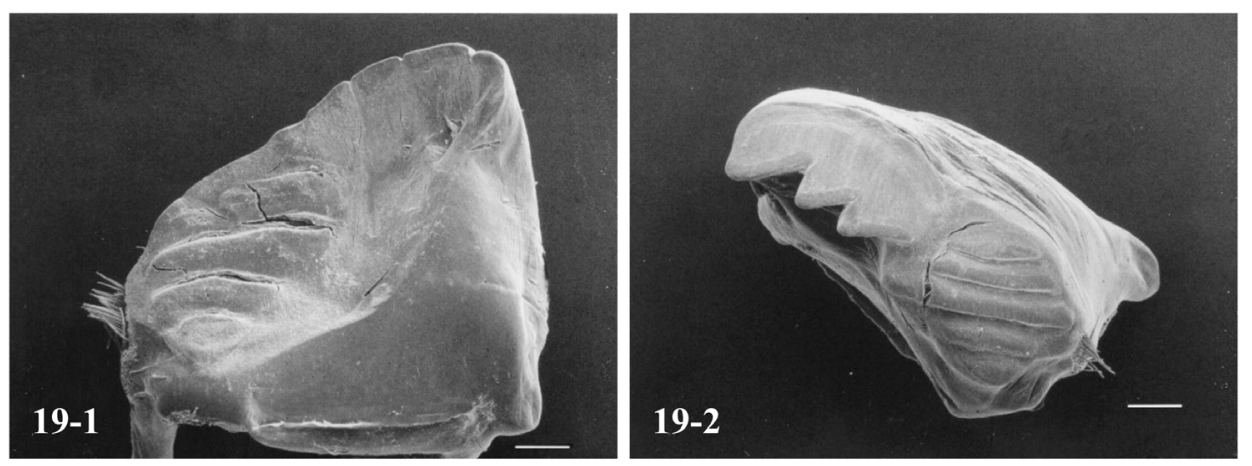

Figure 19. Dnopherula svenhedini (Sjöstedt, 1933) adult mandible, 19-1: left mandible posterior view; 19-2: right mandible lateral view (scale bar $=250 \mu \mathrm{m}$ ).
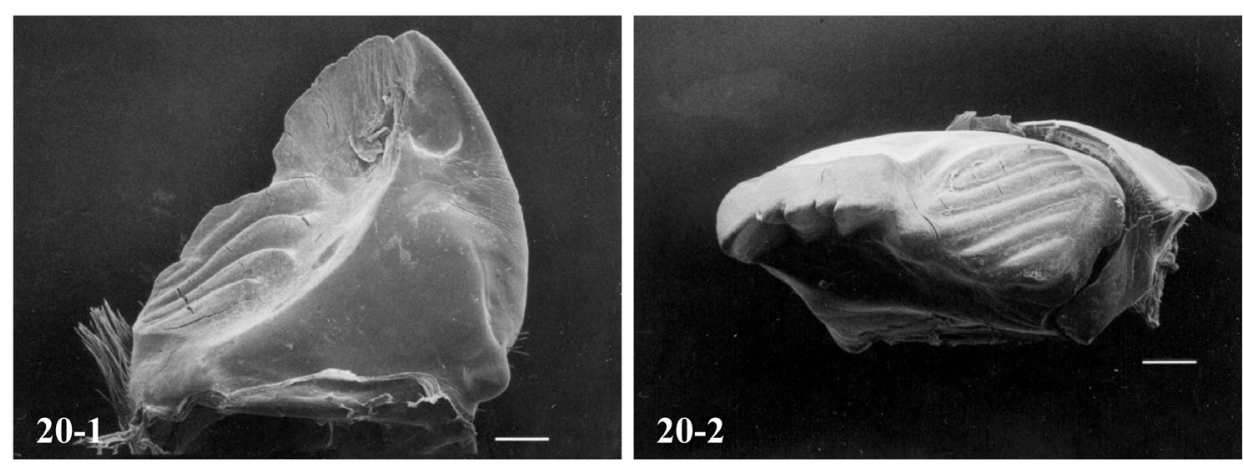

Figure 20. Formosacris koshunensis (Shiraki, 1910) adult mandible, 20-1: left mandible posterior view; 20-2: right mandible lateral view (scale bar $=250 \mu \mathrm{m})$. 


\section{Discussion}

Three types of Mandibles of Grasshoppers to Food adaptation: based on the right mandible of totally 20 species of grasshoppers, there are three types of food adaptation that were identified.

Type 1: Figure 12-1 and Figure 12-2 incisory teeth digitate, distinct; molar area of right mandible with transverse ridge short and longitudinal groove present; longitudinal groove more than $68 \%$ width of molar area, right mandible with molar area longer than wide about 1.3 - 2 times. This type much more matched the description of previous works [4] [8] which they defined as forbivory mandibles. The longitudinal groove is significant for feeding on the leaves, flowers, and buds of broad leaves plant, the forbs accordingly.

Type2: Figure 13-1 and Figure 13-2, incisory teeth indistinct, margin smoothly. Molar area without longitudinal groove, instate, comprise of many long ridges. Rught mandible with molar area is broad and flat, wider than long about $0.8-1.3$ times. This type is close to the definition of [8] and [4] as the graminivorous mandibles, that are good for feeding on herbs.

Type 3: Figure 14-1 and Figure 14-2, incisory area with intercalary teeth distinct; molar area of right mandible with short longitudinal groove, as long as molar area about 0.4 - 0.6 times. Right mandible with molar area is longer than wide about $1.4-2$ times.

The grasshoppers belong to each of three types are list up in Table 1. The grasshop

Table 1. The species numbers and taxa of grasshoppers possessed different mandibular types in Guandaushi forest ecosystem, central Taiwan.

\begin{tabular}{|c|c|c|}
\hline Mandible type & Grasshopper species & Species No. \\
\hline $\begin{array}{c}\text { Type1 } \\
\text { Forbivory feeding }\end{array}$ & $\begin{array}{l}\text { Catantopidae } \\
\text { Traulia ornata ornata } \\
\text { Xenocatantops humilis } \\
\text { Coptacra sp. } 3 \\
\text { Pyrgomorphidae } \\
\text { Atractomorpha sinensis } \\
\text { Eumastacidae } \\
\text { Erianthella formosana }\end{array}$ & 5 \\
\hline $\begin{array}{c}\text { Type } 2 \\
\text { Graminivory feeding }\end{array}$ & $\begin{array}{l}\text { Catantopidae } \\
\text { Oxya podisma } \\
\text { Oxyrrhepes obtusa } \\
\text { Acrididae } \\
\text { Phlaeoba albonema } \\
\text { Phlaeoba formosana } \\
\text { Phlaeoba sinensis } \\
\text { Arcypteridae } \\
\text { Formosacris koshunensis } \\
\text { Dnopherula svenhedini } \\
\text { Oedipodidae } \\
\text { Pternoscirta sauteri } \\
\text { Heteropternis respondens } \\
\text { Trilophidia annulata }\end{array}$ & 10 \\
\hline $\begin{array}{c}\text { Type } 3 \\
\text { Obligate type } \\
\text { Herb-forb feeding }\end{array}$ & $\begin{array}{l}\text { Catantopidae } \\
\text { Stenocatantops splendens } \\
\text { Parapodisma sp. } 1 \\
\text { Parapodisma sp. } 2 \\
\text { Sinopodisma kodamae } \\
\text { Sinopodisma formosana }\end{array}$ & 5 \\
\hline
\end{tabular}


per belonging to type 1 are Traulia ornata ornate, Xenocatantops humilis and Coptacra sp. 3 of Catantopidae; Atractomorpha sinensis of Pygomorphidae and Erianthella formosanas of Eumastacidae. The species belonging to type 2 are Oxya podisma, Oxyrrhepes obtusa of Catantopidae; Phlaeoba albonema, Phlaeoba formosana and Phlaeoba sinensis of Acridae; Formosacris koshunensis and Dnopherula svenhedini of Arcypteridae and Pternoscirta sauteri, Heteropternis respondens and Trilophidia annulata of Oeipodidae. The species belonging to type 3 are Stenocatantops splendens, Parapodisma sp. 1, Parapodisma sp. 2, Sinopodisma kodamae, and Sinopodisma formosana of Catantopidae. In terms of the grasshopper diversity in the forest ecosystem related to the diversity of plant species, the feeding behavior adaptation interact to the food plant types in the subtropical forest based on the morphology of mandible is reasonable and proved.

Based on SEM observations, the mandibles of them could be grouped into three types corresponding to their food plant. The three types were defined according to the morphology of longitudinal groove, the incisory teeth in addition to the central concavity of molar area [7]. Other characters were compared with previous works [4] [8].

The report of Kang et al. [7] emphasizes that the central concavity of mandible is the key character of herbivore grasshoppers. We found similar result that the type 1 for bfeeding, central concavity of molar area surrounded by short ridges; type 2 grass-feeding, the molar surface has long parallel grinding ridges without central concavity; type 3 obligate-feeding, the molar consists of ridges with a central concavity shorter than type 1. It is obvious to define the central concavity of molar area as the diagnostic character for the grasshopper mandible type.

\section{References}

[1] Mulkern, G.B. (1967) Food Selection by Grasshoppers. Annual Review of Entomology, 12, 59-78. https://doi.org/10.1146/annurev.en.12.010167.000423

[2] Chapman, R.F. (1990) Feeding. In: Chapman, R.F. and Joern, A., Eds., Biology of Grasshoppers, A Wiley-Interscience Publication, New Jersey, 40-103.

[3] Gangwere, S.K. (1991) Food Habits and Feeding Behavior of Locusts and Grasshoppers. Department of Biology Sciences, Wayne State University, Detroit, 56 p.

[4] Gangwere, S.K. (1965) The Structural Adaptations of Mouthparts in Orthoptera and Allies. Eos, Revista Espanola de Entomolgia, 41, 67-85.

[5] Bernays, E.A. (1986) Diet-Induced Head Allometry among Foliage-Chewing Insects and Its Importance for Graminivores. Science, 231, 495-497.

https://doi.org/10.1126/science.231.4737.495

[6] Gangwere, S.K., McKinney, J.C., Ernemann, M.A. and Bland, R.G. (1998) Food Selection and Feeding Behavior in Selected Acridoidea (Insecta: Orthoptera) of the Canary Islands, Spain. Journal of Orthoptera Research, 7, 1-21. https://doi.org/10.2307/3503485

[7] Kang, L., Gan, Y. and Li, S. (1999) The Structural Adaptation of Mandibles and Food Specificity in Grasshoppers on Inner Mongolian Grasslands. Journal of Orthoptera Research, 8, 257-269. https://doi.org/10.2307/3503442

[8] Isely, F.B. (1944) Correlation between Mandibular Morphology and Food Specificity in Grasshoppers. Annals of the Entomological Society of America, 37, 47-67. https://doi.org/10.1093/aesa/37.1.47

[9] Yang, J.T. and Chang, Y.L. (1996) A New Genus Taiwanemobius (Orthoptrna: Grylloidea: Gryllidae: Nemobiinae) of Ground Crickets from Taiwan. Journal of Orthoptera Research, 5, 61-64. https://doi.org/10.2307/3503583 
Submit or recommend next manuscript to SCIRP and we will provide best service for you:

Accepting pre-submission inquiries through Email, Facebook, LinkedIn, Twitter, etc. A wide selection of journals (inclusive of 9 subjects, more than 200 journals) Providing 24-hour high-quality service

User-friendly online submission system

Fair and swift peer-review system

Efficient typesetting and proofreading procedure

Display of the result of downloads and visits, as well as the number of cited articles Maximum dissemination of your research work

Submit your manuscript at: http://papersubmission.scirp.org/

Or contact ae@scirp.org 